\title{
Asymmetric Synthesis of Spiro Tetrahydrothiophene-indan-1,3- diones via a Squaramide-Catalyzed Sulfa-Michael/Aldol Domino Reaction
}

\author{
Suruchi Mahajan \\ Pankaj Chauhana \\ Marcus Blümel ${ }^{a}$ \\ Rakesh Puttreddy \\ Kari Rissanen ${ }^{b}$ \\ Gerhard Raabe ${ }^{a}$ \\ Dieter Enders*a \\ a Institute of Organic Chemistry, RWTH Aachen University, \\ Landoltweg 1, 52074 Aachen, Germany \\ enders@rwth-aachen.de \\ b Department of Chemistry, Nanoscience Center, University \\ of Jyvaskyla, 40014 JYU, Finland
}

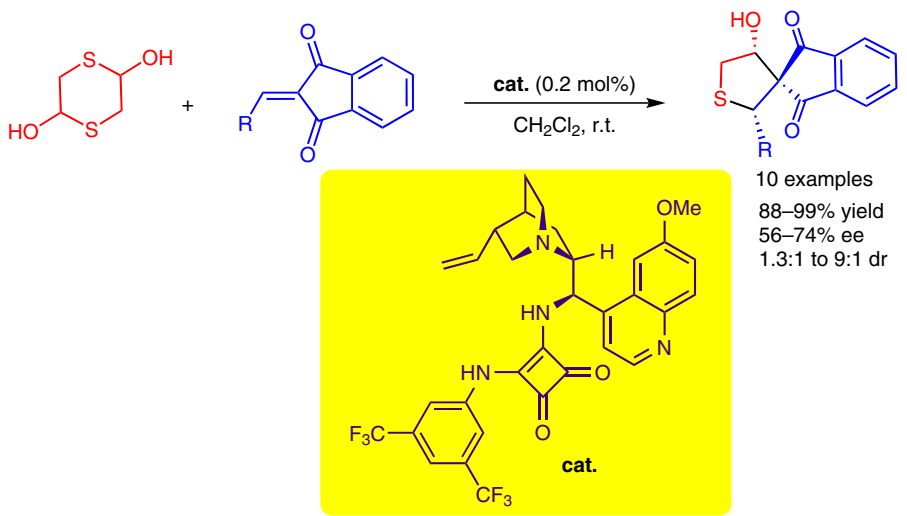

as an essential coenzyme biotin (A) - a water-soluble vitamin involved in important biological functions, ${ }^{4}$ the various penicillins $\mathbf{B}^{5}$ the nucleoside $\mathbf{C}$ showing potent activity against human cytomegalovirus, ${ }^{6}$ and glucosidase inhibitors, such as salacinol $\mathbf{D}^{7}$ (Figure 1 ). Recently, some organocatalytic asymmetric or non-enantioselective domino sulfaMichael/Michael additions or sulfa-Michael/aldol reactions have been developed for the synthesis of the tetrahydrothiophene ring. ${ }^{8}$ These strategies have been extended to the asymmetric synthesis of tetrahydrothiophene bearing spiro oxindoles $^{8 \mathrm{f}}$ and benzodihydropyrane derivatives. ${ }^{8 \mathrm{~m}}$ Other than these spiro oxindoles and benzodihydropyranes, there is another class of important spiro compounds that is, the
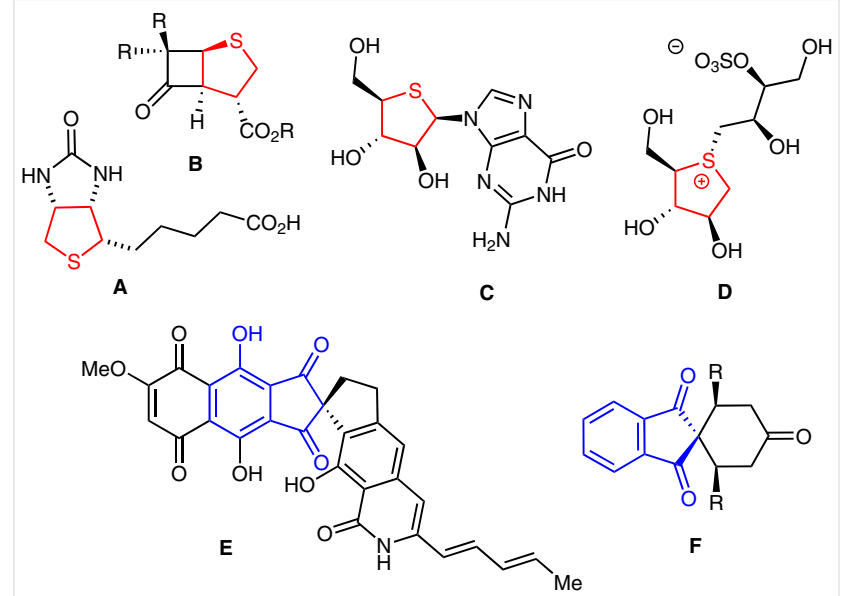

Figure 1 Representative examples of enantiopure bioactive compounds bearing a tetrahydrothiophene or a spiro indan-1,3-dione moiety 
spiro indan-1,3-diones, which are very important building blocks and show several biological activities such as antitumor, antibiotic (E), and anticancer (F) $\operatorname{activity}^{9}$ (Figure 1).

The 2-arylidene-1,3-indandiones are the best known precursors for the synthesis of the spiro indan-1,3-diones. ${ }^{10,11}$ The 2-arylidene-1,3-indandiones were first explored in asymmetric reactions about a decade ago by the Barbas group, ${ }^{11 a}$ but so far only a few asymmetric transformations have been reported on the asymmetric synthesis of spiro indan-1,3-diones using 2-arylidene-1,3-indandiones.

Owing to our interest in achieving squaramide-catalyzed new asymmetric domino reactions, ${ }^{3,11 j, 12}$ we herein report a new asymmetric domino sulfa-Michael/aldol reaction of 1,4-dithiane-2,5-diol with 2-arylidene-1,3-indandiones catalyzed by a low loading of a squaramide, which combine the two important indan-1,3-dione and a tetrahydrothiophene cores in a single structure. ${ }^{13}$
We first started our investigation by screening various bifunctional organocatalysts for the reaction of 1,4-dithiane-2,5-diol (1) with 2-benzylidene-1,3-indandione (2a) in dichloromethane as solvent (Table 1 ). It was observed that the reaction occurs rapidly using all catalysts with excellent yields and good diastereoselectivities. The cinchona derived squaramide catalysts IV-VII (Figure 2 ) bearing a 3,5-( $\left(\mathrm{CF}_{3}\right)_{2}-$ phenyl group directly attached to the squaramide unit provide better ee values than the catalyst I-III bearing a 3,5$\left(\mathrm{CF}_{3}\right)_{2}$-benzyl group (Table 1 , entries $\left.1-7\right)$. The quinidine derived squaramide $\mathbf{V}$ gives the best ee of $58 \%$ (entry 5 ). The squaramide VIII, thioureas IX-XI, cupreine derivatives XIIXIII, and natural cinchona alkaloids XIV-XV (Figure 2) were tested further in order to improve the enantioselectivity, but all these catalysts gave lower ee values than the catalyst V (entries 8-15).<smiles>C=CC1CN2CCC1C2[C@H](Nc1c(NCc2cc(C(F)(F)F)cc(C(F)(F)F)c2)c(=O)c1=O)c1cnc2ccc(OC)cc2c1</smiles><smiles></smiles><smiles>C=CC1CN2CCC1C2[C@H](Nc1c(NCc2cc(C(F)(F)F)cc(C(F)(F)F)c2)c(=O)c1=O)C1=COC=Nc2ccccc21</smiles><smiles></smiles><smiles>C=CC1CN2CCC1C2[C@H](Nc1c(Nc2cc(C(F)(F)F)cc(C(F)(F)F)c2)c(=O)c1=O)c1ccnc2ccc(OC)cc12</smiles><smiles>C=CC1C2CCN(C2C=C)C(Nc2c(Nc3cc(C(F)(F)F)cc(C(F)(F)F)c3)c(=O)c2=O)C1c1ccnc2ccccc12</smiles><smiles>C=CC1CN2CCC1C2[C@H](Nc1c(Nc2cc(C(F)(F)F)cc(C(F)(F)F)c2)c(=O)c1=O)c1ccnc2ccccc12</smiles><smiles>CN(C)[C@H]1CCCC[C@H]1Nc1c(Nc2cc(C(F)(F)F)cc(C(F)(F)F)c2)c(=O)c1=O</smiles><smiles>CN(C)[C@H]1CCCC[C@H]1NC(=S)Nc1cc(C(F)(F)F)cc(C(F)(F)F)c1</smiles><smiles>[X]C1CCCCC1NC(=S)Nc1cc(C(F)(F)F)cc(C(F)(F)F)c1</smiles><smiles>C=CC1CC2CCN1C2C(NC(=S)Nc1cc(C(F)(F)F)cc(C(F)(F)F)c1)c1ccnc2ccc(OC)cc12</smiles><smiles>C=CC1CC2CCN1C2[C@H](O)c1ccnc2ccc(O)cc12</smiles>

XII<smiles>C=CC1CC2CCN1C2[C@H](C=C)c1ccnc2ccc(O)cc12</smiles><smiles>C=CC1CN2CCC1C2[C@H](O)c1ccnc2ccc(OC)cc12</smiles><smiles>C=CC1CN2CCC1C2[C@H](O)c1ccnc2ccccc12</smiles>

$\mathrm{xv}$

Figure 2 Organocatalysts used 
Table 1 Catalyst Screeninga

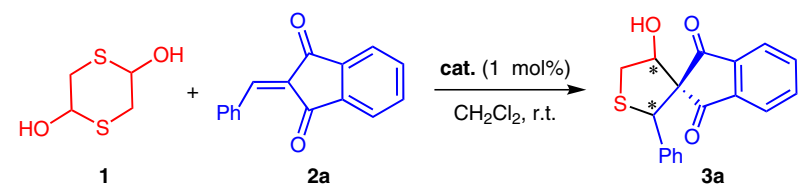

\begin{tabular}{clllc}
\hline Entry & Catalyst & Time (h) & Yield (\%) $^{\mathrm{b}}$ & ee (\%),d \\
\hline 1 & I & 1.0 & 93 & -27 \\
2 & II & 1.5 & 95 & 34 \\
3 & III & 1.5 & 92 & -28 \\
4 & IV & 2.0 & 98 & -51 \\
5 & V & 1.75 & 97 & 58 \\
6 & VI & 2.0 & 97 & -51 \\
7 & VII & 1.75 & 97 & 49 \\
8 & VIII & 1.75 & 97 & 36 \\
9 & IX & 1.5 & 95 & 21 \\
10 & X & 1.5 & 95 & -7 \\
11 & XI & 2.0 & 85 & -20 \\
12 & XII & 1.5 & 93 & -3 \\
13 & XIII & 1.5 & 85 & -9 \\
14 & XIV & 2.0 & 97 & 6 \\
15 & XV & 2.0 & 96 & 7
\end{tabular}

a Reaction conditions: $0.2 \mathrm{mmol}$ of $\mathbf{1}, 0.2 \mathrm{mmol} \mathbf{2 a}, 1 \mathrm{~mol} \%$ of catalyst in $0.5 \mathrm{~mL}$ of $\mathrm{CH}_{2} \mathrm{Cl}_{2}$ at r.t.

${ }^{\mathrm{b}}$ Yield of isolated product after column chromatography.

' Enantiomeric excess of the major diastereomer $(9: 1 \mathrm{dr})$ was determined

by HPLC analysis of the acylated product on a chiral stationary phase.

d Negative sign indicates the ee of the opposite enantiomer.

Further optimizations were carried out by screening different solvents using catalyst $\mathbf{V}$ (Table 2). However, no solvent provided a better enantioselectivity than dichloromethane (Table 2, entries 1-7). The dilution of the reaction concentration led to an enhanced ee value of $67 \%$ when 2.0 $\mathrm{mL}$ of dichloromethane was used (entries 8,9 ). In order to increase the enantioselectivity further, the reaction was carried out at $-20^{\circ} \mathrm{C}$, but a slight drop in the ee value with a lower reaction rate was observed (entry 10). The screening of different additives such as anhydrous $\mathrm{Na}_{2} \mathrm{SO}_{4}, \mathrm{MgSO}_{4}$, and molecular sieves did not increase the enantioselectivity (entries 11-13). Using benzoic acid as an additive led to a slow reaction rate with low yield and enantioselectivity (entry 14), probably due to the protonation of the tertiary amine of the catalyst. It is interesting to note that even a catalyst loading as low as $0.1 \mathrm{~mol} \%$ worked very well without affecting the chemical yield and stereoselectivity of the transformations.

We further evaluated the substrate scope for the domino sulfa-Michael/1,2-addition reaction using $0.2 \mathrm{~mol} \%$ of the catalyst $\mathbf{V}$ (Table 3). The 2-arylidene-1,3-indandiones $\mathbf{2}$ bearing electron neutral groups react efficiently with 1,4dithiane-2,5-diol (1) to provide the corresponding products 3a and $\mathbf{3 b}$ in high yields with good diastereoselectivities and moderate ee values. The 2-arylidene-1,3-indandiones bearing electron-withdrawing nitro and trifluoromethyl groups undergo a rapid sulfa-Michael/1,2-addition reaction in excellent yields and with good $\mathrm{dr}$ of $56 \%$ (3c) and $66 \%$ ee (3d), respectively. The 4-bromo- and 2-chlorophenyl derivatives of $\mathbf{2}$ resulted in a poor diastereomeric ratio with a higher ee of the minor diastereomer 3e,f. This domino sequence also tolerates electron-donating substituents on the phenyl ring to provide the desired products $\mathbf{3 g}$-i in high yield with good $\mathrm{dr}$ and moderate enantioselectivities. The heteroaryl group on $\mathbf{2}$ was found to be less reactive under the optimized reaction conditions, requiring a longer reaction time to afford the corresponding products with good $\mathrm{dr}$ and moderate enantioselectivities.

A gram-scale domino sulfa-Michael/1,2-addition reaction of $\mathbf{1}$ with $\mathbf{2 a}$ also worked very well without any effect on the chemical yields and stereoselectivity of the product (Scheme 1).

Table 2 Reaction Optimization ${ }^{\mathrm{a}}$

\begin{tabular}{|c|c|c|c|c|}
\hline Entry & Solvent (mL) & Time (h) & Yield (\%) & ee $(\%)^{c}$ \\
\hline 1 & $\mathrm{CH}_{2} \mathrm{Cl}_{2}(0.5)$ & 1.5 & 93 & 61 \\
\hline 2 & $\mathrm{ClCH}_{2} \mathrm{CH}_{2} \mathrm{Cl}(0.5)$ & 2.5 & 95 & 58 \\
\hline 3 & $\mathrm{CHCl}_{3}(0.5)$ & 1.5 & 92 & 53 \\
\hline 4 & toluene $(0.5)$ & 3.5 & 98 & 36 \\
\hline 5 & $\operatorname{THF}(0.5)$ & 2.0 & 97 & 39 \\
\hline 6 & MTBE (0.5) & 24 & 94 & 37 \\
\hline 7 & 1,4-dioxane (0.5) & 2.0 & 95 & 41 \\
\hline 8 & $\mathrm{CH}_{2} \mathrm{Cl}_{2}(1.0)$ & 1.5 & 97 & 63 \\
\hline 9 & $\mathrm{CH}_{2} \mathrm{Cl}_{2}(2.0)$ & 1.5 & 97 & 67 \\
\hline $10^{\mathrm{d}}$ & $\mathrm{CH}_{2} \mathrm{Cl}_{2}(2.0)$ & 24 & 95 & 65 \\
\hline $11^{\mathrm{e}}$ & $\mathrm{CH}_{2} \mathrm{Cl}_{2}(2.0)$ & 2.0 & 94 & 66 \\
\hline $12^{f}$ & $\mathrm{CH}_{2} \mathrm{Cl}_{2}(2.0)$ & 2.0 & 94 & 68 \\
\hline $13^{9}$ & $\mathrm{CH}_{2} \mathrm{Cl}_{2}(2.0)$ & 2.0 & 95 & 68 \\
\hline $14^{\mathrm{h}}$ & $\mathrm{CH}_{2} \mathrm{Cl}_{2}(2.0)$ & 96 & 37 & 29 \\
\hline $15^{\mathrm{i}}$ & $\mathrm{CH}_{2} \mathrm{Cl}_{2}(4.0)$ & 2.0 & 96 & 68 \\
\hline $16^{j}$ & $\mathrm{CH}_{2} \mathrm{Cl}_{2}(10.0)$ & 2.5 & 96 & 68 \\
\hline $17^{k}$ & $\mathrm{CH}_{2} \mathrm{Cl}_{2}(10.0)$ & 7.0 & 94 & 68 \\
\hline
\end{tabular}

a Reaction conditions: $0.2 \mathrm{mmol}$ of $\mathbf{1}, 0.2 \mathrm{mmol} \mathbf{2 a}, 1 \mathrm{~mol} \%$ of $\mathbf{V}$ in $0.5 \mathrm{~mL}$ of solvent at r.t.

${ }^{b}$ Yield of isolated product after column chromatography.

c Enantiomeric excess of the major diastereomer (9:1 dr) was determined by HPLC analysis of the acylated product on a chiral stationary phase.

d The reaction was performed at $-20^{\circ} \mathrm{C}$.

e Anhydrous $\mathrm{Na}_{2} \mathrm{SO}_{4}(100 \mathrm{mg})$ was used as an additive.

${ }^{\mathrm{f}}$ Anhydrous $\mathrm{MgSO}_{4}(100 \mathrm{mg})$ was used as an additive.

g MS $5 \AA$ (100 mg) was used as an additive.

h $\mathrm{PhCO}_{2} \mathrm{H}(20 \mathrm{~mol} \%)$ was used as an additive.

i The reaction was performed at $0.4 \mathrm{mmol}$ scale using $0.5 \mathrm{~mol} \%$ of $\mathbf{V}$.

j The reaction was performed at $1.0 \mathrm{mmol}$ scale using $0.2 \mathrm{~mol} \%$ of $\mathbf{V}$.

$\mathrm{k}$ The reaction was performed at $1.0 \mathrm{mmol}$ scale using $0.1 \mathrm{~mol} \%$ of $\mathbf{V}$. 
Table 3 Substrate Scope<smiles>OC1CSC(O)CS1</smiles>

1<smiles>[R]C=C1C(=O)c2ccccc2C1=O</smiles>

$\underset{\mathrm{CH}_{2} \mathrm{Cl}_{2} \text {, r.t. }}{\stackrel{\text { V }(0.2 \mathrm{~mol} \%)}{\longrightarrow}}$

2

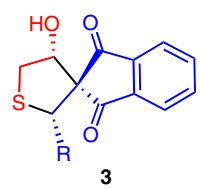

3

\begin{tabular}{llllll}
\hline 3 & $\mathrm{R}$ & Time (h) & ${\text { Yield }(\%)^{\mathrm{b}}}^{\mathrm{d}}$ & $\mathrm{dr}^{\mathrm{c}}$ & ee (\%) \\
\hline a & $\mathrm{Ph}$ & 3.0 & 96 & $9: 1$ & 68 \\
b & 1-naphthyl & 3.0 & 93 & $8: 1$ & 65 \\
c & $4-\mathrm{O}_{2} \mathrm{NC}_{6} \mathrm{H}_{4}$ & 2.0 & 99 & $7.5: 1$ & 56 \\
d & $4-\mathrm{F}_{3} \mathrm{CC}_{6} \mathrm{H}_{4}$ & 1.0 & 96 & $9: 1$ & 66 \\
e & $4-\mathrm{BrC}_{6} \mathrm{H}_{4}$ & 5.0 & 97 & $1.3: 1$ & $68,83^{\mathrm{e}}$ \\
f & 2- $\mathrm{ClC}_{6} \mathrm{H}_{4}$ & 2.0 & 93 & $1.5: 1$ & $72,75^{\mathrm{e}}$ \\
g & $4-\mathrm{MeC}_{6} \mathrm{H}_{4}$ & 2.5 & 98 & $9: 1$ & 71 \\
h & 3- $\mathrm{MeOC}_{6} \mathrm{H}_{4}$ & 3.0 & 88 & $9: 1$ & 60 \\
i & 3,4- $\left(\mathrm{OCH}_{2} \mathrm{O}\right) \mathrm{C}_{6} \mathrm{H}_{3}$ & 4.5 & 96 & $9: 1$ & 74 \\
j & 3-thienyl & 24 & 98 & $9: 1$ & 72
\end{tabular}

a Reaction conditions: $1.0 \mathrm{mmol}$ of $\mathbf{1}, 1.0 \mathrm{mmol} \mathbf{2}, 0.2 \mathrm{~mol} \%$ of $\mathbf{V}$ in $10 \mathrm{~mL}$ of $\mathrm{CH}_{2} \mathrm{Cl}_{2}$ at r.t.

${ }^{b}$ Yield of isolated product after column chromatography.

' Diastereomeric ratio (cis to trans) was determined by HPLC of the acylated product.

${ }^{d}$ Enantiomeric excess of the major diastereomer was determined by HPLC analysis of the acylated product on a chiral stationary phase.

e Enantiomeric excess of the minor diastereomer.

$$
\begin{aligned}
& \text { SO } \\
& \begin{array}{c}
1 \\
0.76 \mathrm{~g}
\end{array} \\
& \text { (5 mmol) } \\
& \text { 2a } \\
& \text { (5 mmol) }
\end{aligned}
$$

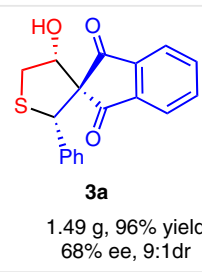

$68 \%$ ee, $9: 1 d r$
Scheme 1 Gram-scale reaction

In order to determine the relative and absolute configuration of the spiro tetrahydrothiophene-indan-1,3-dione products, the corresponding carbamate $\mathbf{4}$ was prepared from 3a. Unfortunately, even after several attempts of crystallization only the racemic mixture crystallized. The X-ray crystallographic analysis revealed the cis-configuration of the product, ${ }^{14}$ which is also in agreement with the NOESY experiment of 4 (Figure 3 ).

The absolute configuration was determined by CD measurements and calculations for the acylated product $\mathbf{3} \mathbf{j}$ ' to be $R, R$. Thus, based on the CIP-rules all the other products 3a-j have $2 ' S, 4^{\prime} R$ configuration (Figure 4, see also Supporting Information).

In conclusion, we have developed a new asymmetric domino sulfa-Michael/aldol reaction of 2-arylidene-1,3-indandiones with 1,4-dithiane-2,5-diol catalyzed by only 0.2 $\mathrm{mol} \%$ of a squaramide. This domino transformation provides a rapid access to the tetrahydrothiophene bearing

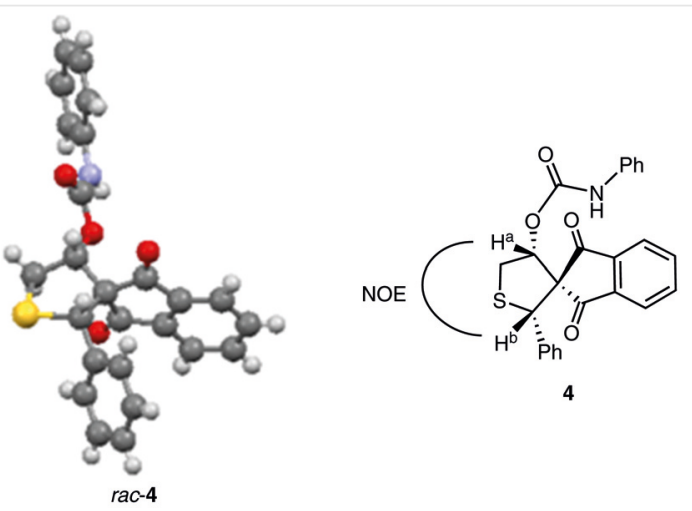

Figure 3 Determination of the relative configuration by X-ray structure analysis and NOESY measurement of carbamate 4

spiro indane-1,3-dione derivatives in excellent yields with moderate to good diastereoselectivities and moderate enantioselectivities.
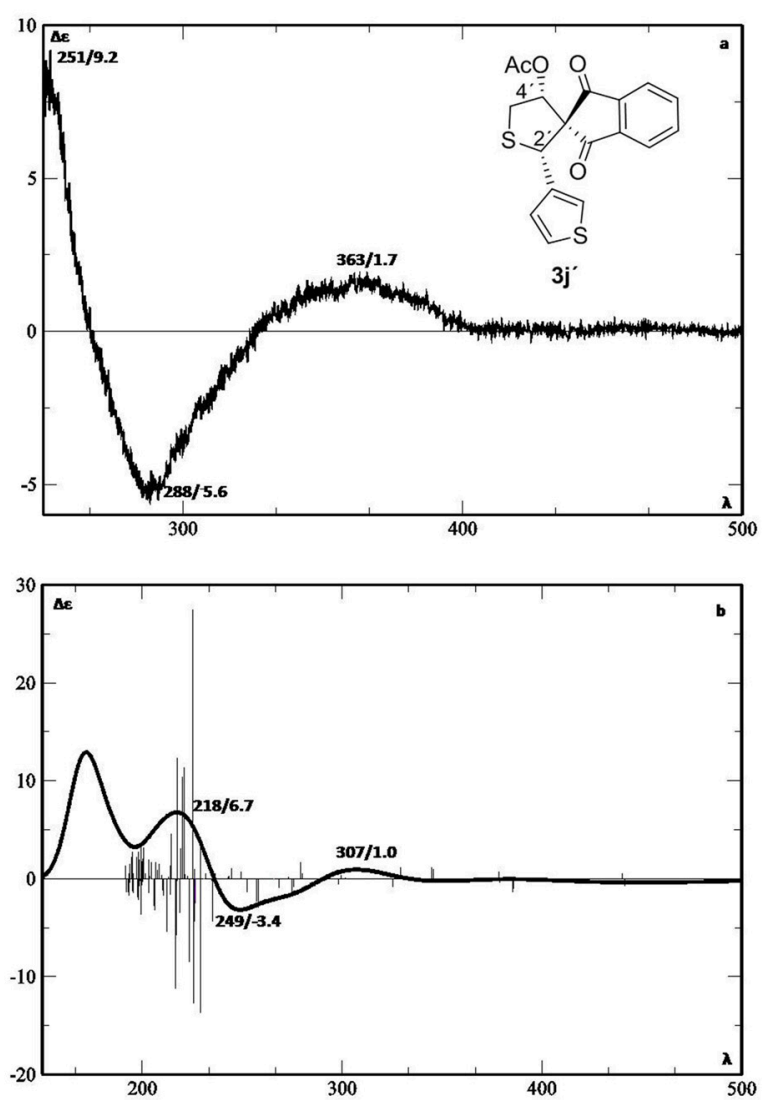

Figure 4 Determination of the absolute configuration of $\mathbf{3} \mathbf{j}^{\prime}$ by comparison of its experimental electronic circular dichroism (ECD) spectrum measured at room temperature in acetonitrile (top, a) with its calculated counterpart Boltzmann-averaged from the ECD spectra of two conformers (bottom, b). $\Delta \varepsilon$ is in $1000 \mathrm{~cm}^{2} \mathrm{~mol}^{-1}$ and $\lambda$ in $\mathrm{nm}$. For details, see the electronic supplementary material. 
All reactions were performed in oven-dried glassware. Analytical TLC was performed using SIL G-25 UV254 from Machery \& Nagel and visualized with ultraviolet radiation at $254 \mathrm{~nm} .{ }^{1} \mathrm{H}$ and ${ }^{13} \mathrm{C}$ NMR spectra were recorded in $\mathrm{CDCl}_{3}$ at ambient temperature on Varian Innova 600 instrument. Chemical shifts for ${ }^{1} \mathrm{H}$ NMR and ${ }^{13} \mathrm{C}$ NMR spectra for the major cis-isomer are reported in parts per million (ppm), with coupling constants given in Hertz (Hz). Standard abbreviations are used for denoting spin multiplicities. Mass spectra were recorded on SSQ7000 spectrometer from Finnigan at $70 \mathrm{eV}$, whereas HRMS data (ESI) were collected using a ThermoFisher Scientific LTQ-Orbitrap XL apparatus. IR spectra were recorded on a PerkinElmer Spectrum 100 FT-IR spectrometer. Elemental analyses were performed with a Vario EL elemental analyzer. Analytical HPLC was carried out either on a Hewlett-Packard 1050 series instrument or Agilent 1100 instrument using chiral stationary phases. Optical rotation values were measured on a PerkinElmer 241 polarimeter and melting points using a Büchi 510 apparatus (both on the diastereometic mixture of $\mathbf{3 a - j}$ ).

Unless specified, the starting materials and reagents were purchased directly from the commercial suppliers and used without further purification. All solvents used as reaction media were distilled before use. The 2-arylidene-1,3-indandiones $\mathbf{2}^{11 \mathrm{a}}$ and the catalysts $\mathbf{I}-\mathbf{V I I I},{ }^{15}$ $\mathbf{X},{ }^{16} \mathbf{X I},{ }^{17}$ and $\mathbf{X I I}-\mathbf{X I I I}{ }^{18}$ were synthesized using known literature procedures. For HPLC analyses the racemic samples of $\mathbf{3 a} \mathbf{a}-\mathbf{j}$ were synthesized using DBU as catalyst. For the determination of the enantiomeric excess by HPLC the products $\mathbf{3 a}-\mathbf{j}$ were acylated in $\mathrm{CH}_{2} \mathrm{Cl}_{2}$ using $\mathrm{Ac}_{2} \mathrm{O}$, pyridine, and DMAP.

\section{Compounds 3a-j; General Procedure}

In an oven dried round-bottom flask, a solution of the squaramide catalyst $\mathbf{V}(0.2 \mathrm{~mol} \%)$ and the corresponding 2-arylidene-1,3-indandione $2(1.0 \mathrm{mmol})$ in $\mathrm{CH}_{2} \mathrm{Cl}_{2}(10 \mathrm{~mL})$ was stirred at r.t. After $5 \mathrm{~min}$, the 1,4-dithiane-2,5-diol $(\mathbf{1} ; 1.0 \mathrm{mmol})$ was added and the stirring was continued until the complete consumption of the reactants was observed by TLC. Then the crude mixture was purified by flash chromatography on silica gel using a gradient of $n$-hexane/EtOAc (9:1 to 3:1) to afford the desired product $\mathbf{3}$ (Table 3 ).

\section{4'-Hydroxy-2'-phenyl-4',5'-dihydro-2' $H$-spiro[indene-2,3'-thio- phene]-1,3-dione (3a)}

Yield: $299 \mathrm{mg}$ (96\%); colorless solid; $\mathrm{mp} 115-116^{\circ} \mathrm{C}$; $[\alpha]_{\mathrm{D}}{ }^{24}+66.6$ $\left(c=0.5, \mathrm{CHCl}_{3}\right)$.

HPLC: Chiralpak IC column; $230 \mathrm{~nm}, n$-heptane/EtOH (9:1), 0.70 $\mathrm{mL} / \mathrm{min}, t_{\mathrm{R}}=6.69 \min$ (major), $8.37 \mathrm{~min}$ (minor); $9: 1 \mathrm{dr}$; 68\% ee.

IR (capillary): 3473, 3024, 2938, 2649, 2321, 2185, 2099, 1974, 1891, 1738, 1699, 1589, 1493, 1445, 1353, 1246, 1163, 1077, 1028, 930, $889,844,754,696 \mathrm{~cm}^{-1}$.

${ }^{1} \mathrm{H}$ NMR $\left(600 \mathrm{MHz}, \mathrm{CDCl}_{3}\right): \delta=7.83-7.81(\mathrm{~m}, 1 \mathrm{H}, \mathrm{ArH}), 7.69-7.63(\mathrm{~m}$, $3 \mathrm{H}, \mathrm{ArH}), 7.27-7.26(\mathrm{~m}, 2 \mathrm{H}, \mathrm{ArH}), 7.06-7.02(\mathrm{~m}, 3 \mathrm{H}, \mathrm{ArH}) 5.10(\mathrm{~s}, 1$ $\mathrm{H}, \mathrm{SCHAr}), 5.04-5.00\left(\mathrm{~m}, 1 \mathrm{H}, \mathrm{HOCHCH}_{2}\right), 3.85-3.82\left(\mathrm{~m}, 1 \mathrm{H}, \mathrm{CH}_{2}\right)$, $3.36\left(\mathrm{dd}, J=10.0,7.0 \mathrm{~Hz}, 1 \mathrm{H}, \mathrm{CH}_{2}\right), 2.53(\mathrm{~d}, J=6.6 \mathrm{~Hz}, 1 \mathrm{H}, \mathrm{OH})$.

${ }^{13} \mathrm{C}$ NMR $\left(151 \mathrm{MHz}, \mathrm{CDCl}_{3}\right): \delta=200.9,198.2,143.3,142.4,136.6$, 136.1, 135.5, 135.1, 128.8 (2C), 128.4, 128.3 (2 C), 123.0 (2 C), 80.1, $69.3,53.2,36.2$.

MS (CI, methane): $m / z=311.1[\mathrm{M}+\mathrm{H}]^{+}$.

HRMS (ESI): $m / z[\mathrm{M}+\mathrm{Na}]^{+}$calcd for $\mathrm{C}_{18} \mathrm{H}_{14} \mathrm{O}_{3} \mathrm{SNa}$ : 333.0556; found: 333.0563 .

\section{4'-Hydroxy-2'-(naphthalen-1-yl)-4',5'-dihydro-2'H-spiro[indene-} 2,3'-thiophene]-1,3-dione (3b)

Yield: $335 \mathrm{mg}$ (93\%); light yellow solid; $\mathrm{mp} 85-87^{\circ} \mathrm{C} ;[\alpha]_{\mathrm{D}}{ }^{24}+82.2$ $\left(c=0.5, \mathrm{CHCl}_{3}\right)$.

HPLC: Chiralpak IC column; $230 \mathrm{~nm}, n$-heptane/EtOH (9.7:0.3), 1.0 $\mathrm{mL} / \mathrm{min}, t_{\mathrm{R}}=10.11$ (major), $13.66 \mathrm{~min}$ (minor); 8:1 dr; 65\% ee.

IR (capillary): 3426, 3055, 2937, 2653, 2319, 2098, 1994, 1915, 1737 , $1698,1590,1505,1436,1353,1256,1157,1075,909,866,751 \mathrm{~cm}^{-1}$.

${ }^{1} \mathrm{H}$ NMR $\left(600 \mathrm{MHz}, \mathrm{CDCl}_{3}\right): \delta=7.84(\mathrm{~d}, J=7.5 \mathrm{~Hz}, 1 \mathrm{H}, \mathrm{ArH}), 7.78(\mathrm{~s}, 1$ $\mathrm{H}, \mathrm{ArH}), 7.70-7.35(\mathrm{~m}, 6 \mathrm{H}, \mathrm{ArH}), 7.40-7.35(\mathrm{~m}, 3 \mathrm{H}, \mathrm{ArH}), 5.30(\mathrm{~s}, 1 \mathrm{H}$, SCHAr), 5.09-5.06 (m, $\left.1 \mathrm{H}, \mathrm{HOCHCH}_{2}\right), 3.91-3.88\left(\mathrm{~m}, 1 \mathrm{H}, \mathrm{CH}_{2}\right), 3.41$ (dd, $\left.J=10.0,7.0 \mathrm{~Hz}, 1 \mathrm{H}, \mathrm{CH}_{2}\right), 2.31(\mathrm{~s}, 1 \mathrm{H}, \mathrm{OH})$.

${ }^{13} \mathrm{C}$ NMR (151 MHz, $\mathrm{CDCl}_{3}$ ): $\delta=200.7,198.0,143.2,142.3,136.0$, 135.4, 133.0, 132.8, 132.6, 128.1, 128.0, 127.9, 127.4, 126.2, 126.1, 126.0, 123.0, 122.9, 80.2, 69.1, 53.1, 36.2.

MS (ESI): $m / z=399.1[\mathrm{M}+\mathrm{K}]^{+}$.

HRMS (ESI): $m / z$ [M] calcd for $\mathrm{C}_{22} \mathrm{H}_{16} \mathrm{O}_{3} \mathrm{~S}$ : 360.0815; found: 360.0815 .

\section{4'-Hydroxy-2'-(4-nitrophenyl)-4',5'-dihydro-2'H-spiro[indene- 2,3'-thiophene]-1,3-dione (3c)}

Yield: $352 \mathrm{mg}$ (99\%); colorless solid; $\mathrm{mp} 148-150{ }^{\circ} \mathrm{C} ;[\alpha]_{\mathrm{D}}{ }^{24}+90.0$ $\left(c=0.5, \mathrm{CHCl}_{3}\right)$.

HPLC: Chiralpak IB column; $230 \mathrm{~nm}, n$-heptane/i-PrOH (9:1), 0.50 $\mathrm{mL} / \mathrm{min}, t_{\mathrm{R}}=27.59$ (major), $32.11 \mathrm{~min}$ (minor); 7.5:1 dr; 56\% ee.

IR (capillary): 3469, 3077, 2925, 2855, 2650, 2456, 2320, 2103, 1993 , 1901, 1736, 1699, 1594, 1518, 1434, 1344, 1255, 1166, 1075, 908 , $835,757,693 \mathrm{~cm}^{-1}$.

${ }^{1} \mathrm{H}$ NMR $\left(600 \mathrm{MHz}, \mathrm{CDCl}_{3}\right): \delta=7.94-7.89(\mathrm{~m}, 3 \mathrm{H}, \mathrm{ArH}), 7.75-7.72(\mathrm{~m}$, $3 \mathrm{H}, \mathrm{ArH}), 7.51-7.42$ (m, $2 \mathrm{H}, \mathrm{ArH}), 5.19$ (s, $1 \mathrm{H}, \mathrm{SCHAr}), 5.04-5.01$ (m, $\left.1 \mathrm{H}, \mathrm{HOCHCH}_{2}\right), 3.87-3.84\left(\mathrm{~m}, 1 \mathrm{H}, \mathrm{CH}_{2}\right), 3.37(\mathrm{dd}, J=10.0,6.8 \mathrm{~Hz}, 1 \mathrm{H}$, $\mathrm{CH}_{2}$ ), 2.28 (br s, $1 \mathrm{H}, \mathrm{OH}$ ).

${ }^{13} \mathrm{C}$ NMR (151 MHz, $\left.\mathrm{CDCl}_{3}\right): \delta=200.0,197.4,147.6,143.1,142.9$, 142.1, 136.5, 135.9, 129.8 (2C), 123.3 (2 C), 123.1 (2 C), 80.4, 68.8, 51.6, 36.2 .

MS (EI): $m / z=355.1[\mathrm{M}]^{+}$.

HRMS (ESI): $m / z$ [M + Na] ${ }^{+}$calcd for $\mathrm{C}_{18} \mathrm{H}_{13} \mathrm{NO}_{5} \mathrm{SNa}$ : 378.0407; found: 378.0408 .

\section{4'-Hydroxy-2'-[4-(trifluoromethyl)phenyl]-4',5'-dihydro-2' $H$ - spiro[indene-2,3'-thiophene]-1,3-dione (3d)}

Yield: $365 \mathrm{mg}$ (96\%); light yellow solid; mp $97-99{ }^{\circ} \mathrm{C} ;[\alpha]_{\mathrm{D}}{ }^{24}+50.4$ $\left(c=0.5, \mathrm{CHCl}_{3}\right)$.

HPLC: Chiralpak IC column; $254 \mathrm{~nm}, n$-heptane/EtOH (9:1), $1 \mathrm{~mL} / \mathrm{min}$, $t_{\mathrm{R}}=3.52 \mathrm{~min}$ (major), $4.70 \mathrm{~min}$ (minor); 9:1 dr; 66\% ee.

IR (capillary): 3471, 3060, 2940, 2642, 2322, 2185, 2106, 2000, 1939 , 1739, 1695, 1591, 1512, 1407, 1323, 1259, 1115, 1067, 894, 841, 768, $708,666 \mathrm{~cm}^{-1}$.

${ }^{1} \mathrm{H}$ NMR $\left(600 \mathrm{MHz}, \mathrm{CDCl}_{3}\right): \delta=7.86-7.85(\mathrm{~m}, 1 \mathrm{H}, \mathrm{ArH}), 7.72-7.69(\mathrm{~m}$, $3 \mathrm{H}, \mathrm{ArH}), 7.42(\mathrm{~d}, J=8.2 \mathrm{~Hz}, 2 \mathrm{H} \mathrm{ArH}), 7.32(\mathrm{~d}, J=8.1 \mathrm{~Hz}, 2 \mathrm{H}, \mathrm{ArH})$, 5.15 (s, $1 \mathrm{H}, \mathrm{SCHAr}), 5.04-5.01\left(\mathrm{~m}, 1 \mathrm{H}, \mathrm{HOCHCH}_{2}\right), 3.86-3.82(\mathrm{~m}, 1 \mathrm{H}$, $\mathrm{CH}_{2}$ ), 3.36 (dd, $J=9.9,6.9 \mathrm{~Hz}, 1 \mathrm{H}, \mathrm{CH}_{2}$ ), 2.48 (br s, $1 \mathrm{H}, \mathrm{OH}$ ).

${ }^{13} \mathrm{C}$ NMR (151 MHz, $\left.\mathrm{CDCl}_{3}\right): \delta=200.4,197.7,143.0,142.2,139.5$, 136.3 (2 C), 135.7 (2 C), 129.1 (2 C), 125.2 (2 C), 123.0 (2 C), 80.3, 68.9, $52.0,36.1$.

MS (ESI): $m / z=417.0[\mathrm{M}+\mathrm{K}]^{+}$.

Anal. Calcd for $\mathrm{C}_{19} \mathrm{H}_{13} \mathrm{~F}_{3} \mathrm{O}_{3} \mathrm{~S}$ : C, 60.31; H, 3.46. Found: $\mathrm{C}, 59.95 ; \mathrm{H}, 3.62$. 
2'-(4-Bromophenyl)-4'-hydroxy-4',5'-dihydro-2'H-spiro[indene2,3'-thiophene]-1,3-dione (3e)

Yield: $378 \mathrm{mg}$ (97\%); colorless solid; $\mathrm{mp} 140-142{ }^{\circ} \mathrm{C} ;[\alpha]_{\mathrm{D}}{ }^{24}-26.0$ $\left(c=0.5, \mathrm{CHCl}_{3}\right)$.

HPLC: Chiralpak IC column; $230 \mathrm{~nm}, n$-heptane/i-PrOH (9:1), 0.7 $\mathrm{mL} / \mathrm{min}, t_{\mathrm{R}}=15.03 \mathrm{~min}$ (major), $16.59 \mathrm{~min}$ (minor) and $t_{\mathrm{R}}=8.91 \mathrm{~min}$ (major), $9.63 \mathrm{~min}$ (minor); 1.3:1 dr; 68\% ee (major diastereomer), 83\% ee (minor diastereomer).

IR (capillary): 3445, 3082, 2932, 2704, 2321, 2109, 1998, 1736, 1695, 1589, 1465, 1436, 1350, 1257, 1166, 1131, 1074, 1027, 940, 895, 836, $758,672 \mathrm{~cm}^{-1}$.

${ }^{1} \mathrm{H}$ NMR $\left(600 \mathrm{MHz}, \mathrm{CDCl}_{3}\right): \delta=7.99-7.90(\mathrm{~m}, 2 \mathrm{H})_{\text {both }}, 7.76-7.65(\mathrm{~m}, 3$ $\mathrm{H}, \mathrm{ArH})_{\text {both }}, 7.25-7.17(\mathrm{~m}, 2 \mathrm{H})_{\text {both }}, 6.98-6.90(\mathrm{~m}, 1 \mathrm{H}, \mathrm{ArH})_{\text {both }}, 5.64(\mathrm{~s}$, $1 \mathrm{H}, \mathrm{SCHAr})_{\text {minor }}, 5.54(\mathrm{~s}, 1 \mathrm{H}, \mathrm{SCHAr})_{\text {major }}, 5.03-4.99(\mathrm{~m}, 1 \mathrm{H}$, $\left.\mathrm{HOCHCH}_{2}\right)_{\text {major }}, 4.90-4.88\left(\mathrm{~m}, 1 \mathrm{H}, \mathrm{HOCHCH}_{2}\right)_{\text {minor }}, 3.83$ (dd, $J=10.1$, $\left.8.8 \mathrm{~Hz}, 1 \mathrm{H}, \mathrm{CH}_{2}\right)_{\text {major }}, 3.72\left(\mathrm{dd}, J=10.9,4.7 \mathrm{~Hz}, 1 \mathrm{H}, \mathrm{CH}_{2}\right)_{\text {minor }}, 3.55(\mathrm{~d}$, $J=4.1 \mathrm{~Hz}, 1 \mathrm{H}, \mathrm{OH})_{\text {minor }}, 3.41-3.37\left(\mathrm{~m}, 1 \mathrm{H}, \mathrm{CH}_{2}\right)_{\text {both }}, 2.80(\mathrm{~d}, J=6.6 \mathrm{~Hz}$, $1 \mathrm{H}, \mathrm{OH})_{\text {major }}$.

${ }^{13} \mathrm{C}$ NMR $\left(151 \mathrm{MHz}, \mathrm{CDCl}_{3}\right): \delta$ (both isomers) $=201.2,199.7,198.2$, 197.7, 142.7 (2 C), 142.5, 142.0, 136.2, 136.0, $135.9135 .8,135.7$ (2 C) 133.0, 132.5, 132.4129 .5 (2 C), 127.4 (2 C), 124.9, 124.5, 123.4, 123.0 (2 C), 80.66, 80.5, 68.0, 67.3, 53.4, 51.4, 38.5, 37.2.

MS (ESI): $m / z=429.0[\mathrm{M}+\mathrm{K}]^{+}$.

Anal. Calcd for $\mathrm{C}_{18} \mathrm{H}_{13} \mathrm{BrO}_{3} \mathrm{~S}$ : C, 55.54; H, 3.37. Found: C, 55.57; H, 3.38.

\section{2'-(2-Chlorophenyl)-4'-hydroxy-4',5'-dihydro-2'H-spiro[indene-} 2,3'-thiophene]-1,3-dione (3f)

Yield: $320 \mathrm{mg}$ (93\%); colorless solid; $\mathrm{mp} 141-142{ }^{\circ} \mathrm{C} ;[\alpha]_{\mathrm{D}}{ }^{24}+2.4$ $\left(c=0.5, \mathrm{CHCl}_{3}\right)$.

HPLC: Chiralpak IC column; $230 \mathrm{~nm}, n$-heptane/i-PrOH (9:1), 1.0 $\mathrm{mL} / \mathrm{min}, t_{\mathrm{R}}=9.64 \mathrm{~min}$ (major), $11.66 \mathrm{~min}$ (minor) and $t_{\mathrm{R}}=5.99 \mathrm{~min}$ (major), $6.81 \mathrm{~min}$ (minor); 1.5:1 dr; 72\% ee (major diastereomer), 75\% ee (minor diastereomer).

IR (capillary): 3478, 3083, 2937, 2702, 2320, 2110, 1931, 1737, 1695, 1589, 1468, 1437, 1349, 1256, 1165, 1129, 1072, 1040, 940, 894869 , $841,794,757,692 \mathrm{~cm}^{-1}$.

${ }^{1} \mathrm{H}$ NMR(600 MHz, $\left.\mathrm{CDCl}_{3}\right): \delta=7.99-7.88(\mathrm{~m}, 2 \mathrm{H}, \mathrm{ArH})_{\text {both }}, 7.76-7.63$ $(\mathrm{m}, 3 \mathrm{H}, \mathrm{ArH})_{\text {both }}, 7.18(\mathrm{dd}, J=14.9,7.1 \mathrm{~Hz}, 1 \mathrm{H}, \mathrm{ArH})_{\text {both }}, 6.96-7.03(\mathrm{~m}$, $2 \mathrm{H}, \mathrm{ArH})_{\text {both }}, 5.68(\mathrm{~s}, 1 \mathrm{H}, \mathrm{CH}, \mathrm{SCHAr})_{\text {minor }}, 5.55(\mathrm{~s}, 1 \mathrm{H}, \mathrm{CH}, \mathrm{SCHAr})_{\text {major }}$, 5.05-5.01 (m, $\left.1 \mathrm{H}, \mathrm{HOCHCH}_{2}\right)_{\text {major }}, 4.88-4.86\left(\mathrm{~m}, 1 \mathrm{H}, \mathrm{HOCHCH}_{2}\right)$, 3.85-3.82 (m, $\left.1 \mathrm{H}, \mathrm{CH}_{2}\right)_{\text {major }}$, 3.77-3.74 (m, $\left.1 \mathrm{H}, \mathrm{ArH}, \mathrm{CH}_{2}\right)_{\text {minor }}$, $3.67(\mathrm{~d}$, $J=3.6 \mathrm{~Hz}, 1 \mathrm{H}, \mathrm{OH})_{\text {minor }}, 3.38-3.35\left(\mathrm{~m}, 1 \mathrm{H}, \mathrm{CH}_{2}\right)_{\text {both }}, 2.83(\mathrm{~d}, J=6.5 \mathrm{~Hz}$, $1 \mathrm{H}, \mathrm{OH})_{\text {major }}$.

${ }^{13} \mathrm{C}$ NMR $\left(151 \mathrm{MHz}, \mathrm{CDCl}_{3}\right): \delta$ (both isomers) $=201.3,199.9,198.2$, 197.6, 142.7, 142.5, 142.5, 141.9, 136.2, 136.0, 135.9, 135.8, 134.0, $133.9,133.8,133.7,132.7,132.2,129.2,129.2,129.1,129.0,126.8$, 126.8, 123.4, 123.4, 123.0, 122.9, 80.7, 80.5, 68.0, 67.4, 51.1, 48.9, 38.8, 37.0.

MS (ESI): $m / z=383.0[\mathrm{M}+\mathrm{K}]^{+}$.

Anal. Calcd for $\mathrm{C}_{18} \mathrm{H}_{13} \mathrm{ClO}_{3} \mathrm{~S}$ : C, 62.70; $\mathrm{H}, 3.80$. Found: C, 62.94; $\mathrm{H}, 3.66$.

4'-Hydroxy-2'-(4-methylphenyl)-4',5'-dihydro-2'H-spiro[indene2,3'-thiophene]-1,3-dione (3g)

Yield: $319 \mathrm{mg}$ (98\%); yellow solid; mp $57-59{ }^{\circ} \mathrm{C} ;[\alpha]_{\mathrm{D}}{ }^{24}+40.4(c=0.5$, $\left.\mathrm{CHCl}_{3}\right)$.
HPLC: Chiralpak IC column; $230 \mathrm{~nm}, n$-heptane/i-PrOH (9:1), 0.70 $\mathrm{mL} / \mathrm{min}, t_{\mathrm{R}}=11.23 \mathrm{~min}$ (major), $18.53 \mathrm{~min}$ (minor); 9:1 dr; 71\% ee.

IR (capillary): 3427, 3024, 2933, 2649, 2319, 2108, 1904, 1736, 1697 , 1591, 1509, 1439, 1350, 1254, 1169, 1074, 899, 821, 780, 748, 669 $\mathrm{cm}^{-1}$.

${ }^{1} \mathrm{H}$ NMR $\left(600 \mathrm{MHz}, \mathrm{CDCl}_{3}\right): \delta=7.84(\mathrm{~d}, J=7.0 \mathrm{~Hz}, 1 \mathrm{H}, \mathrm{ArH}), 7.71-7.64$ $(\mathrm{m}, 3 \mathrm{H}, \mathrm{ArH}), 7.15$ (d, J=8.1 Hz, $2 \mathrm{H}, \operatorname{ArH}), 6.85(\mathrm{~d}, J=8.0 \mathrm{~Hz}, 2 \mathrm{H}$, ArH), 5.08 (s, $1 \mathrm{H}, \mathrm{SCHAr}), 5.02-4.99\left(\mathrm{~m}, 1 \mathrm{H}, \mathrm{HOCHCH}_{2}\right), 3.83-3.80$ (m, $1 \mathrm{H}, \mathrm{CH}_{2}$ ), 3.35 (dd, $J=10.0,7.0 \mathrm{~Hz}, 1 \mathrm{H}, \mathrm{CH}_{2}$ ), 2.45 (br s, $1 \mathrm{H}, \mathrm{OH}$ ), $2.12\left(\mathrm{~s}, 3 \mathrm{H}, \mathrm{CH}_{3}\right)$.

${ }^{13} \mathrm{C}$ NMR (151 MHz, $\left.\mathrm{CDCl}_{3}\right): \delta=200.8,198.2,143.3,142.3,137.9$, $135.9,135.3,131.9,128.9,128.8$ (2C), 128.5 (2C), 122.9, 80.1, 69.2, $52.8,36.0,20.9$.

MS (CI, methane): $m / z=325.0[\mathrm{M}+\mathrm{H}]^{+}$.

HRMS (ESI): $m / z[M]^{+}$calcd for $\mathrm{C}_{19} \mathrm{H}_{16} \mathrm{NO}_{3} \mathrm{~S}$ : 324.0815; found: 324.0813 .

4'-Hydroxy-2'-(3-methoxyphenyl)-4',5'-dihydro-2'H-spiro[indene2,3'-thiophene]-1,3-dione (3h)

Yield: $299 \mathrm{mg}$ (88\%); light yellow wax; $[\alpha]_{\mathrm{D}}{ }^{24}+55.2\left(c=0.5, \mathrm{CHCl}_{3}\right)$.

HPLC: Chiralpak IA column; $254 \mathrm{~nm}, n$-heptane/EtOH (7:3), 0.70 $\mathrm{mL} / \mathrm{min}, t_{\mathrm{R}}=10.29$ (major), $10.99 \mathrm{~min}$ (minor); 9:1 dr; 60\% ee.

IR (capillary): 3425, 3015, 2939, 2836, 2649, 2323, 2097, 1982, 1867 , $1694,1588,1485,1444,1350,1253,1154,1045,918,767,689 \mathrm{~cm}^{-1}$.

${ }^{1} \mathrm{H} \mathrm{NMR}\left(600 \mathrm{MHz}, \mathrm{CDCl}_{3}\right): \delta=7.82-7.80(\mathrm{~m}, 1 \mathrm{H}, \mathrm{ArH}), 7.69-7.68(\mathrm{~m}$, $1 \mathrm{H}, \mathrm{ArH}), 7.65-7.63$ (m, $1 \mathrm{H}, \mathrm{ArH}), 6.93-6.91$ (m, $2 \mathrm{H}, \mathrm{ArH}), 6.87-6.86$ $(\mathrm{m}, 1 \mathrm{H}, \mathrm{ArH}), 6.79(\mathrm{~d}, J=7.7 \mathrm{~Hz}, 1 \mathrm{H}, \mathrm{ArH}), 6.55(\mathrm{dd}, J=8.2,2.6 \mathrm{~Hz}, 1$ $\mathrm{H}, \mathrm{ArH}), 5.07$ (s, $1 \mathrm{H}, \mathrm{SCHAr}), 5.02-4.99\left(\mathrm{~m}, 1 \mathrm{H}, \mathrm{HOCHCH}_{2}\right), 3.84-3.80$ $\left(\mathrm{m}, 1 \mathrm{H}, \mathrm{CH}_{2}\right), 3.64\left(\mathrm{~s}, 3 \mathrm{H}, \mathrm{CH}_{3}\right), 3.34\left(\mathrm{dd}, J=9.9,7.0 \mathrm{~Hz}, 1 \mathrm{H}, \mathrm{CH}_{2}\right), 2.74$ (br s, $1 \mathrm{H}, \mathrm{OH}$ ).

${ }^{13} \mathrm{C}$ NMR (151 MHz, $\mathrm{CDCl}_{3}$ ): $\delta=200.8,198.0,159.2,143.2,142.3$, 136.6, 135.9, 135.3, 129.1, 122.9, 122.8, 120.9, 114.3, 113.6, 80.0, 69.1, 55.1, 53.0, 35.9 .

MS (ESI): $m / z=363.1[\mathrm{M}+\mathrm{Na}]^{+}$.

HRMS (ESI): $m / z$ [M] calcd for $\mathrm{C}_{19} \mathrm{H}_{16} \mathrm{O}_{4} \mathrm{~S}$ : 340.0764 ; found: 340.0775 .

\section{2'-(Benzo[d][1,3]dioxol-5-yl)-4'-hydroxy-4',5'-dihydro-2'H- spiro[indene-2,3'-thiophene]-1,3-dione (3i)}

Yield: $341 \mathrm{mg}$ (96\%); yellow solid; $\mathrm{mp} 72-74^{\circ} \mathrm{C}$; $[\alpha]_{\mathrm{D}}{ }^{24}+92.4(c=0.5$, $\mathrm{CHCl}_{3}$ ).

HPLC: Chiralpak IC column; $230 \mathrm{~nm}, n$-heptane/EtOH (9.7:0.3), 1.0 $\mathrm{mL} / \mathrm{min}, t_{\mathrm{R}}=19.42$ (major), $21.37 \mathrm{~min}$ (minor); 9:1 dr; 74\% ee.

IR (capillary): 3449, 3014, 2939, 2646, 2321, 2181, 2087, 1993, 1949, 1875, 1738, 1700, 1591, 1488, 1439, 1360, 1245, 1076, 1035, 925 , $789,755,666 \mathrm{~cm}^{-1}$.

${ }^{1} \mathrm{H}$ NMR $\left(600 \mathrm{MHz}, \mathrm{CDCl}_{3}\right): \delta=7.86-7.84(\mathrm{~m}, 1 \mathrm{H}), 7.77-7.70(\mathrm{~m}, 3 \mathrm{H}$, ArH), 6.88 (d, $J=1.8 \mathrm{~Hz}, 1 \mathrm{H}), 6.68$ (dd, $J=8.1,1.8 \mathrm{~Hz}, 1 \mathrm{H}, \mathrm{ArH}), 6.45$ (d, $J=8.1 \mathrm{~Hz}, 1 \mathrm{H}, \mathrm{ArH}), 5.80-5.78\left(\mathrm{~m}, 2 \mathrm{H}, \mathrm{OCH}_{2} \mathrm{O}\right), 5.03(\mathrm{~s}, 1 \mathrm{H}$, SCHAr), 4.98-4.95 (m, $\left.1 \mathrm{H}, \mathrm{HOCHCH}_{2}\right), 3.82-3.78\left(\mathrm{~m}, 1 \mathrm{H}, \mathrm{CH}_{2}\right), 3.33$ (dd, $\left.J=10.0,7.0 \mathrm{~Hz}, 1 \mathrm{H}, \mathrm{CH}_{2}\right), 2.34(\mathrm{~s}, 1 \mathrm{H}, \mathrm{OH})$.

${ }^{13} \mathrm{C}$ NMR (151 MHz, $\left.\mathrm{CDCl}_{3}\right): \delta=200.7,198.1,147.4,147.3,143.3$, $142.4,136.0,135.4,128.7,123.0,122.9,122.2,109.2,107.7,101.0$, 79.9, 69.1, 52.9, 36.0.

MS (ESI): $m / z=393.0[\mathrm{M}+\mathrm{K}]^{+}$.

HRMS (ESI): $m / z$ [M] $]^{+}$calcd for $\mathrm{C}_{19} \mathrm{H}_{14} \mathrm{O}_{5} \mathrm{~S}$ : 354.0556; found: 354.0560 . 


\section{4'-Hydroxy-2'-(thiophen-3-yl)-4',5'-dihydro-2'H-spiro[indene- 2,3'-thiophene]-1,3-dione (3j)}

Yield: $309 \mathrm{mg}$ (98\%); yellow solid; mp $98-100{ }^{\circ} \mathrm{C} ;[\alpha]_{D}{ }^{24}+60.6(c=0.5$, $\mathrm{CHCl}_{3}$ ).

HPLC: Chiralpak IC column; $254 \mathrm{~nm}, n$-heptane/i-PrOH (9.5:0.5), 1 $\mathrm{mL} / \mathrm{min}, t_{\mathrm{R}}=12.21 \mathrm{~min}$ (major), $17.35 \mathrm{~min}$ (minor); 9:1 dr; 72\% ee.

IR (capillary): 3478, 3414, 3110, 3024, 2935, 2649, 2324, 2180, 2098, 1976, 1951, 1871, 1737, 1696, 1589, 1438, 1353, 1249, 1143, 1075, $935,900,835,775,700 \mathrm{~cm}^{-1}$.

${ }^{1} \mathrm{H}$ NMR $\left(600 \mathrm{MHz}, \mathrm{CDCl}_{3}\right): \delta=7.82-7.81(\mathrm{~m}, 1 \mathrm{H}, \mathrm{ArH}), 7.72-7.66(\mathrm{~m}$, $3 \mathrm{H}, \operatorname{ArH}), 7.12(\mathrm{~d}, J=2.5 \mathrm{~Hz}, 1 \mathrm{H}, \mathrm{ArH}), 6.96(\mathrm{dd}, J=5.0,3.1 \mathrm{~Hz}, \mathrm{ArH})$, 6.80-6.79 (m, $1 \mathrm{H}, \operatorname{ArH}), 5.13(\mathrm{~s}, 1 \mathrm{H}, \mathrm{SCHAr}), 4.99-4.96(\mathrm{~m}, 1 \mathrm{H}$, $\left.\mathrm{HOCHCH}_{2}\right), 3.81-3.78\left(\mathrm{~m}, 1 \mathrm{H}, \mathrm{CH}_{2}\right), 3.32\left(\mathrm{dd}, J=9.9,7.1 \mathrm{~Hz}, 1 \mathrm{H}, \mathrm{CH}_{2}\right.$ ), 2.87 (br s, $1 \mathrm{H}, \mathrm{OH})$.

${ }^{13} \mathrm{C}$ NMR (151 MHz, $\left.\mathrm{CDCl}_{3}\right): \delta=201.0,198.3,143.3,142.4,136.2(2 \mathrm{C})$, $135.5,127.5,126.0,124.3,123.0$ (2 C), 79.8, 68.7, 48.0, 36.0.

MS (ESI): $m / z=339.0[\mathrm{M}+\mathrm{Na}]^{+}$.

Anal. Calcd for $\mathrm{C}_{16} \mathrm{H}_{12} \mathrm{O}_{3} \mathrm{~S}_{2}$ : C, 60.74; H, 3.82. Found: C, 60.54; H, 3.83.

\section{Acylation of 3; General Procedure}

To an oven dried round-bottom flask were added sequentially a solution of 3 (0.5 mmol) in $\mathrm{CH}_{2} \mathrm{Cl}_{2}(5.0 \mathrm{~mL}), \mathrm{Ac}_{2} \mathrm{O}$ (1.0 mmol), DMAP (20 $\mathrm{mol} \%$ ), and pyridine $(1.0 \mathrm{mmol}$ ) and the reaction mixture was stirred for $30 \mathrm{~min}$ at r.t. Then the crude mixture was purified by flash chromatography on silica gel using a gradient of $n$-hexane/EtOAc (9:1 to $4: 1)$ to afford the desired product.

\section{1,3-Dioxo-2'-phenyl-1,3,4',5'-tetrahydro-2'H-spiro[indene-2,3'- thiophen]-4'-yl Acetate (3a') \\ Yield: $156 \mathrm{mg}$ (89\%); colorless wax.}

IR (capillary): 3060, 2946, 1746, 1705, 1593, 1492, 1446, 1365, 1258 , $1218,1043,928,895,841,801,761,699,655 \mathrm{~cm}^{-1}$.

${ }^{1} \mathrm{H}$ NMR $\left(600 \mathrm{MHz}, \mathrm{CDCl}_{3}\right): \delta=7.87-7.86(\mathrm{~m}, 1 \mathrm{H}, \mathrm{ArH}), 7.73-7.68(\mathrm{~m}$, $3 \mathrm{H}, \mathrm{ArH}$ ), 7.27-7.26 (m, $2 \mathrm{H}, \mathrm{ArH}), 7.06-7.03$ (m, $3 \mathrm{H}, \mathrm{ArH}), 5.74$ (dd, $\left.J=9.8,7.3 \mathrm{~Hz}, 1 \mathrm{H}, \mathrm{CHCH}_{2}\right), 5.12(\mathrm{~s}, 1 \mathrm{H}, \mathrm{SCHAr}), 3.76(\mathrm{t}, J=9.8 \mathrm{~Hz}, 1 \mathrm{H}$, $\mathrm{CH}_{2}$ ), 3.59 (dd, $\left.J=9.8,7.3 \mathrm{~Hz}, 1 \mathrm{H}, \mathrm{CH}_{2}\right), 1.76\left(\mathrm{~s}, 3 \mathrm{H}, \mathrm{CH}_{3} \mathrm{CO}\right.$ ).

${ }^{13} \mathrm{C}$ NMR $\left(151 \mathrm{MHz}, \mathrm{CDCl}_{3}\right): \delta=198.8,196.3,169.4,142.7,141.9$, 136.0, 135.5, 134.1, 128.7 (2 C), 128.3, 128.2 (2 C), 123.0 (2 C), 78.8, $67.0,52.4,32.6,20.4$.

MS (EI): $m / z=352.2[\mathrm{M}]^{+}$.

HRMS (ESI): $m / z[\mathrm{M}+\mathrm{Na}]^{+}$calcd for $\mathrm{C}_{20} \mathrm{H}_{16} \mathrm{O}_{4} \mathrm{SNa}$ : 375.0662; found: 375.0669 .

\section{1,3-Dioxo-2'-(thiophen-3-yl)-1,3,4',5'-tetrahydro-2'H-spiro[in- dene-2,3'-thiophen]-4'-yl Acetate (3j') \\ Yield: $171 \mathrm{mg}$ (96\%); colorless wax.}

IR (capillary): 3101, 1745, 1705, 1592, 1435, 1359, 1219, 1043, 898, $835,772 \mathrm{~cm}^{-1}$.

${ }^{1} \mathrm{H} \mathrm{NMR}\left(600 \mathrm{MHz}, \mathrm{CDCl}_{3}\right): \delta=7.89-7.87(\mathrm{~m}, 1 \mathrm{H}, \mathrm{ArH}), 7.78-7.72(\mathrm{~m}$, $3 \mathrm{H}, \mathrm{ArH}), 7.14-7.03(\mathrm{~m}, 1 \mathrm{H}, \mathrm{ArH}), 6.95(\mathrm{dd}, J=5.0,3.0 \mathrm{~Hz}, 1 \mathrm{H}, \mathrm{ArH})$, 6.79 (dd, $J=5.0,1.2 \mathrm{~Hz}, 1 \mathrm{H}, \mathrm{ArH}), 5.69$ (dd, $J=9.8,7.3 \mathrm{~Hz}, 1 \mathrm{H}$, $\mathrm{CHCH}_{2}$ ), 5.16 (s, $\left.1 \mathrm{H}, \mathrm{SCHAr}\right), 3.73-3.68\left(\mathrm{~m}, 1 \mathrm{H}, \mathrm{CH}_{2}\right), 3.54$ (dd, $J=9.8$, $\left.7.3 \mathrm{~Hz}, 1 \mathrm{H}, \mathrm{CH}_{2}\right), 1.74\left(\mathrm{~s}, 3 \mathrm{H}, \mathrm{CH}_{3} \mathrm{CO}\right)$.

${ }^{13} \mathrm{C}$ NMR $\left(151 \mathrm{MHz}, \mathrm{CDCl}_{3}\right): \delta=198.7,196.3,169.3,142.6,141.8$, $136.1,135.5,135.2,127.3,125.9,124.5,123.0$ (2 C), 78.5, 66.4, 47.4, $32.5,20.4$.
MS (ESI): $m / z=397.2[\mathrm{M}+\mathrm{K}]^{+}$.

HRMS (ESI): $m / z$ [M + Na $]^{+}$calcd for $\mathrm{C}_{18} \mathrm{H}_{14} \mathrm{O}_{4} \mathrm{~S}_{2} \mathrm{Na}$ : 381.0226; found: 381.0216 .

1,3-Dioxo-2'-phenyl-1,3,4',5'-tetrahydro-2'H-spiro[indene-2,3'thiophen]-4'-yl Phenylcarbamate (4)

To an oven dried round-bottom flask were added sequentially a solution of 3a $(0.5 \mathrm{mmol})$ in $\mathrm{CH}_{2} \mathrm{Cl}_{2}(5.0 \mathrm{~mL}), \mathrm{N}$-methylimidazole $(20$ $\mathrm{mol} \%)$, and phenyl isocyanate $(1.0 \mathrm{mmol})$ and the reaction mixture was stirred for $22 \mathrm{~h}$ at r.t. Then the crude mixture was purified by flash chromatography on silica gel using a gradient of $n$-hexane/EtOAc (9:1 to 4:1) to afford the desired product 4; yield: $185 \mathrm{mg}$ (86\%); grey solid; mp $156-158{ }^{\circ} \mathrm{C}$.

IR (capillary): 3331, 1737, 1704, 1598, 1532, 1443, 1315, 1258, 1212 , $1159,1060,931,897,753,694 \mathrm{~cm}^{-1}$.

${ }^{1} \mathrm{H}$ NMR $\left(600 \mathrm{MHz}, \mathrm{CDCl}_{3}\right): \delta=7.89-7.86(\mathrm{~m}, 1 \mathrm{H}, \mathrm{ArH}), 7.74-7.67(\mathrm{~m}$, $3 \mathrm{H}, \mathrm{ArH}$ ), 7.36-7.22 (m, $6 \mathrm{H}, \mathrm{ArH}), 7.09-7.02$ (m, $4 \mathrm{H}, \mathrm{ArH}), 6.30$ (s, 1 $\mathrm{H}, \mathrm{NH}), 5.87-5.84\left(\mathrm{~m}, 1 \mathrm{H}, \mathrm{CHCH}_{2}\right), 5.17$ (s, $\left.1 \mathrm{H}, \mathrm{SCHAr}\right), 3.89-3.85(\mathrm{~m}$, $1 \mathrm{H}, \mathrm{CH}_{2}$ ), 3.64 (dd, $J=9.7,7.5 \mathrm{~Hz}, 1 \mathrm{H}, \mathrm{CH}_{2}$ ).

${ }^{13} \mathrm{C}$ NMR $\left(151 \mathrm{MHz}, \mathrm{CDCl}_{3}\right): \delta=198.7,196.6,142.7,142.1,136.0$, 135.9, 135.8, 135.6, 134.2, 129.1, 129.0, 128.7, 128.4 (2 C), 128.2 (3 C), 123.4, 123.2, 123.0, $118.656 .1,52.8,38.1,32.9$.

MS (ESI): $m / z=452.1[\mathrm{M}+\mathrm{Na}]^{+}$.

HRMS (ESI): $m / z[\mathrm{M}+\mathrm{Na}]^{+}$calcd for $\mathrm{C}_{25} \mathrm{H}_{19} \mathrm{NO}_{4} \mathrm{SNa}$ : 452.0927; found: 452.0934 .

\section{Acknowledgment}

We gratefully thank the European Research Council for funding this project with an ERC Advanced Grant 320493 'DOMINOCAT'.

\section{Supporting Information}

Supporting information for this article is available online http://dx.doi.org/10.1055/s-0035-1560412. Included are copies of the ${ }^{1} \mathrm{H}$ and ${ }^{13} \mathrm{C}$ NMR spectra $\mathbf{3 a}-\mathbf{j}, \mathbf{3 a}$ ', 3j' and $\mathbf{4}$, NOSEY of $\mathbf{3 a}$ ' and $\mathbf{4 a}$, and HPLC data of $\mathbf{3} \mathbf{a}^{\prime}-\mathbf{j}$ ', and CD measurement data.

\section{References}

(1) For selected reviews on organocatalytic domino/cascade reactions, see: (a) Enders, D.; Grondal, C.; Hüttl, M. R. M. Angew. Chem. Int. Ed. 2007, 46, 1570. (b) Yu, X.; Wang, W. Org. Biomol. Chem. 2008, 6, 2037. (c) Grondal, C.; Jeanty, M.; Enders, D. Nat. Chem. 2010, 2, 167. (d) Moyano, A.; Rios, R. Chem. Rev. 2011, 111, 4703. (e) Albrecht, Ł.; Jiang, H.; Jørgensen, K. A. Angew. Chem. Int. Ed. 2011, 50, 8492. (f) Grossmann, A.; Enders, D. Angew. Chem. Int. Ed. 2012, 51, 314. (g) Pellissier, H. Adv. Synth. Catal. 2012, 354, 237. (h) Lu, L.-Q.; Chen, J.-R.; Xiao, W.-J. Acc. Chem. Res. 2012, 45, 1278. (i) Goudedranche, S.; Raimondi, W.; Bugaut, X.; Constantieux, T.; Bonne, D.; Rodriguez, J. Synthesis 2013, 45, 1909. (j) Volla, M. R.; Atodiresei, I.; Rueping, M. Chem. Rev. 2014, 114, 2390.

(2) For reviews on squaramide catalysts, see: (a) Alemán, J.; Parra, A.; Jiang, H.; Jørgensen, K. A. Chem. Eur. J. 2011, 17, 6890. (b) Storer, R. I.; Aciro, C.; Jones, L. H. Chem. Soc. Rev. 2011, 40, 2330. (c) Chauhan, P.; Mahajan, S.; Kaya, U.; Hack, D.; Enders, D. Adv. Synth. Catal. 2015, 357, 253. 
(3) (a) Loh, C. C. J.; Hack, D.; Enders, D. Chem. Commun. 2013, 49, 10230. (b) Loh, C. C. J.; Chauhan, P.; Hack, D.; Lehmann, C.; Enders, D. Adv. Synth. Catal. 2014, 356, 3181.

(4) (a) De Clercq, P. J. Chem. Rev. 1997, 97, 1755. (b) Zempleni, J.; Wijeratne, S. S. K.; Hassan, Y. I. Biofactors 2009, 35, 36.

(5) Johnson, J. W.; Evanoff, D. P.; Savard, M. E.; Lange, G.; Ramadhar, T. R.; Assoud, A.; Taylor, N. J.; Dmitrienko, G. I. J. Org. Chem. 2008, 73, 6970 .

(6) Yoshimura, Y.; Watanabe, M.; Satoh, H.; Ashida, N.; Ijichi, K.; Sakata, S.; Machida, H.; Matsuda, A. J. Med. Chem. 1997, 40, 2177.

(7) (a) Yoshikawa, M.; Murakami, T.; Shimada, H.; Matsuda, H.; Yamahara, J.; Tanabe, G.; Muraoka, O. Tetrahedron Lett. 1997, 38, 8367. (b) Yuasa, H.; Takada, J.; Hashimoto, H. Bioorg. Med. Chem. Lett. 2001, 11, 1137. (c) Yoshikawa, M.; Morikawa, T.; Matsuda, H.; Tanabe, G.; Muraoka, O. Bioorg. Med. Chem. 2002, 10, 1547. (d) Matsuda, H.; Morikawa, T.; Yoshikawa, M. Pure Appl. Chem. 2002, 74, 1301.

(8) For a recent review, see: (a) Chauhan, P.; Mahajan, S.; Enders, D. Chem. Rev. 2014, 114, 8807. For recent asymmetric syntheses, see: (b) Brandau, S.; Maerten, E.; Jørgensen, K. A. J. Am. Chem. Soc. 2006, 128, 14986. (c) Baricordi, N.; Benetti, S.; De Risi, C.; Fogagnolo, M.; Pollini, G. P.; Zanirato, V. Lett. Org. Chem. 2009, 6, 593. (d) Luo, G.; Zhang, S.; Duan, W.; Wang, W. Tetrahedron Lett. 2009, 50, 2946. (e) Yu, C.; Zhang, Y.; Song, A.; Ji, Y.; Wang, W. Chem. Eur. J. 2011, 17, 770. (f) Duan, S.-W.; Li, Y.; Liu, Y.-Y.; Zou, Y.-Q.; Shi, D.-Q.; Xiao, W. Chem. Commun. 2012, 5160. (g) Ling, J.-B.; Su, Y.; Zhu, H.-L.; Wang, G.-Y.; Xu, P.-F. Org. Lett. 2012, 14, 1090. (h) Su, Y.; Ling, J.-B.; Zhang, S.; Xu, P.-F. J. Org. Chem. 2013, 78, 11053. (i) Meninno, S.; Croce, G.; Lattanzi, A. Org. Lett. 2013, 15, 3436. (j) Huang, Y.; Zheng, C.; Chai, Z.; Zhao, G. Adv. Synth. Catal. 2014, 356, 579. (k) Liang, J.-J.; Pan, J.-Y.; Xu, D.-C.; Xie, J.W. Tetrahedron Lett. 2014, 55, 6335. (1) Li, Y.-H.; Zhao, B.-L.; Gao, Y.; Du, D.-M. Tetrahedron: Asymmetry 2014, 25, 1513. (m) Zhao, B.-L.; Liu, L.; Du, D.-M. Eur. J. Org. Chem. 2014, 7850. For recent non-enantioselective syntheses, see: (n) Zhong, Y.; Ma, S.; Li, B.; Jiang, X.; Wang, R. J. Org. Chem. 2015, 80, 6870. (o) Hu, Y.-J.; Wang, X.-B.; Li, S.-Y.; Xie, S.-S.; Wang, K. D. G.; Kong, L.-Y. Tetrahedron Lett. 2015, 56, 105. (p) Bharkavi, C.; Kumar, S. V.; Perumal, S. Synlett 2015, 26, 1665.

(9) (a) Evans, P. A.; Brandt, T. A. Tetrahedron Lett. 1996, 37, 1367. (b) Clive, D. L. J.; Kong, X.; Paul, C. C. Tetrahedron 1996, 52, 6085. (c) Nicolaou, K. C.; Montagnon, T.; Vassilikogiannakis, G. Chem. Commun. 2002, 2478. (d) Nicolaou, K. C.; Montagnon, T.; Vassilikogiannakis, G.; Mathison, C. J. N. J. Am. Chem. Soc. 2005, 127, 8872. (e) Pizzirani, D.; Roberti, M.; Grimaudo, S.; Cristina, A. D.; Pipitone, R. M.; Tolomeo, M.; Recanatini, M. J. Med. Chem. 2009, 52, 6936.
(10) For initial non-enantioselective syntheses, see: (a) Ramachary, D. B.; Barbas, C. F. III Chem. Eur. J. 2004, 10, 5323. (b) Ramachary, D. B.; Chowdari, N. S.; Barbas, C. F. III Synlett 2003, 1910.

(11) For stereoselective reactions, see: (a) Ramachary, D. B.; Anebouselvy, K.; Chowdari, N. S.; Barbas, C. F. III J. Org. Chem. 2004, 69, 5838. (b) Ramachary, D. B.; Venkaiah, C.; Krishna, P. M. Chem. Commun. 2012, 48, 2252. (c) Hu, F.; Wei, Y.; Shi, M. Tetrahedron 2012, 68, 7911. (d) Kuan, H.-H.; Chien, C.-H.; Chen, K. Org. Lett. 2013, 15, 2880. (e) Das, U.; Tsai, Y.-L.; Lin, W. Org. Biomol. Chem. 2013, 11, 44. (f) Anwar, S.; Li, S.-M.; Chen, K. Org. Lett. 2014, 16, 2993. (g) Chang, Y.-P.; Gurubrahamam, R.; Chen, K. Org. Lett. 2015, 17, 2908. (h) Zhang, Y.-Y.; Gurubrahamam, R.; Chen, K. Adv. Synth. Catal. 2015, 357, 2457. (i) Duan, J.; Cheng, J.; Li, P. Org. Chem. Front. 2015, 2, 1048. (j) Blümel, M.; Chauhan, P.; Vermeeren, C.; Dreier, A.; Lehmann, C.; Enders, D. Synthesis 2015, 47, 3618.

(12) (a) Hahn, R.; Raabe, G.; Enders, D. Org. Lett. 2014, 16, 3636. (b) Chauhan, P.; Urbanietz, G.; Raabe, G.; Enders, D. Chem. Commun. 2014, 50, 6853. (c) Urbanietz, G.; Atodiresei, I.; Enders, D. Synthesis 2014, 46, 1261. (d) Chauhan, P.; Mahajan, S.; Loh, C. C. J.; Raabe, G.; Enders, D. Org. Lett. 2014, 16, 2954. (e) Loh, C. C. J.; Chauhan, P.; Hack, D.; Lehmann, C.; Enders, D. Adv. Synth. Catal. 2014, 356, 3181. (f) Blümel, M.; Chauhan, P.; Hahn, R.; Raabe, G.; Enders, D. Org. Lett. 2014, 16, 6012. (g) Mahajan, S.; Chauhan, P.; Loh, C. C. J.; Uzungelis, S.; Raabe, G.; Enders, D. Synthesis 2015, 47, 1024. (h) Chauhan, P.; Mahajan, S.; Raabe, G.; Enders, D. Chem. Commun. 2015, 51, 2270.

(13) During the final stage of our work, an enantioselective synthesis of trans-configured spiro tetrahydrothiophene-indan-1,3diones via a thiourea-catalyzed sulfa-Michael/Michael domino reaction was reported: Duan, J.; Cheng, J.; Li, B.; Qi, F.; Li, P. Eur. J. Org. Chem. 2015, 6130.

(14) CCDC-1437740 (for rac-4) contains the supplementary crystallographic data for this paper. These data can be obtained free of charge at www.ccdc.cam.ac.uk/conts/retrieving.html or from the Cambridge Crystallographic Data Centre, 12, Union Road, Cambridge CB2 1EZ, UK; fax: +44 1223336 033; E-mail: deposit@ccdc.cam.ac.uk.

(15) (a) Malerich, J. P.; Hagihara, K.; Rawal, V. H. J. Am. Chem. Soc. 2008, 130, 14416. (b) Zhu, Y.; Malerich, J. P.; Rawal, V. H. Angew. Chem. Int. Ed. 2010, 49, 153.

(16) Nie, S.-Z.; Hu, Z.-P.; Xuan, Y.-N.; Wang, J.-J.; Li, X.-M.; Yan, M. Tetrahedron: Asymmetry 2010, 21, 2055.

(17) Benedek, V.; Varga, S.; Csámpai, A.; Soós, T. Org. Lett. 2005, 7, 1967.

(18) Li, H.; Wang, Y.; Tang, L.; Deng, L. J. Am. Chem. Soc. 2004, 126, 9906. 